
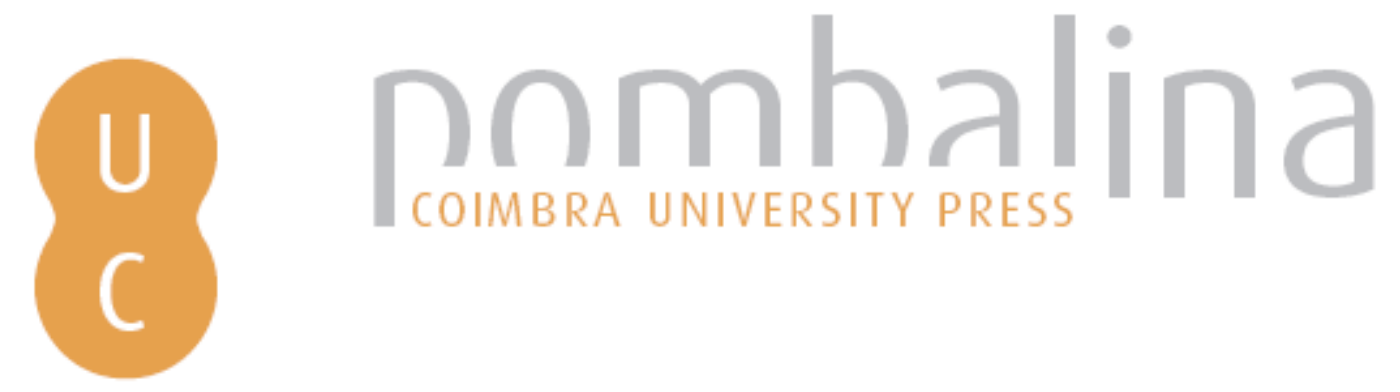

\title{
Espécies vegetaismetalotolerantes e sua potencial utilização na prospeção biogeoquímica e recuperação ambiental (minas de Fragas do Cavalo, Oleiros, Portugal central)
}

\author{
Autor(es): $\quad$ Pratas, J.; Favas, P. J. C.; Conde, L.
}

Publicado por: Imprensa da Universidade de Coimbra

URL persistente:

URI:http://hdl.handle.net/10316.2/31464

DOI:

DOI:http://dx.doi.org/10.14195/978-989-26-0531-9_30

Accessed : $\quad$ 26-Apr-2023 15:09:31

A navegação consulta e descarregamento dos títulos inseridos nas Bibliotecas Digitais UC Digitalis, UC Pombalina e UC Impactum, pressupõem a aceitação plena e sem reservas dos Termos e Condições de Uso destas Bibliotecas Digitais, disponíveis em https://digitalis.uc.pt/pt-pt/termos.

Conforme exposto nos referidos Termos e Condições de Uso, o descarregamento de títulos de acesso restrito requer uma licença válida de autorização devendo o utilizador aceder ao(s) documento(s) a partir de um endereço de IP da instituição detentora da supramencionada licença.

Ao utilizador é apenas permitido o descarregamento para uso pessoal, pelo que o emprego do(s) título(s) descarregado(s) para outro fim, designadamente comercial, carece de autorização do respetivo autor ou editor da obra.

Na medida em que todas as obras da UC Digitalis se encontram protegidas pelo Código do Direito de Autor e Direitos Conexos e demais legislação aplicável, toda a cópia, parcial ou total, deste documento, nos casos em que é legalmente admitida, deverá conter ou fazer-se acompanhar por este aviso.

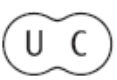





\title{
ESPÉCIES VEGETAIS METALOTOLERANTES E SUA POTENCIAL UTILIZAÇÃO NA PROSPECÇÃO BIOGEOQUÍMICA E RECUPERAÇÃO AMBIENTAL (MINAS DE FRAGAS DO CAVALO, OLEIROS, PORTUGAL CENTRAL)
}

\author{
METALOTOLERANT PLANT SPECIES AND THEIR POTENTIAL \\ IN BIOGEOCHEMICAL PROSPECTING AND ENVIRONMENTAL \\ RESTORATION (FRAGAS DO CAVALO OLD MINE, OLEIROS, \\ CENTRAL PORTUGAL)
}

Resumo - A prospecção biogeoquímica e o controlo ambiental de áreas fortemente poluídas pressupóem uma correta seleção do material biológico a amostrar e uma interpretação dos resultados analíticos que tenha em conta os mecanismos fisiológicos de adaptação das plantas e, fundamentalmente, os fenómenos pedogeoquímicos. Neste trabalho, caracterizaram-se as espécies presentes relativamente à sua capacidade de acumularem metais pesados e metaloides num ambiente com importantes contaminaçóes de W, As e algum Cu, na antiga área mineira de Fragas do Cavalo (Oleiros, Castelo Branco, Portugal Central). Essa caracterização permitiu definir algumas espécies que poderão ser selecionadas como bioindicadoras, ou seja, capazes de detectar a poluição presente no solo, tendo em vista a sua aplicação à prospecção mineira, bem como possibilidade da sua utilização na revegetação dado serem espécies metalotolerantes capazes de suportar a tensão geoquímica imposta por tais condiçóes.

Palavras-chave - Bioacumulação, bioindicação, metais pesados, arsénio, revegetação

Abstract - Biogeochemical prospecting and the environmental control in highly polluted areas presume the correct selection of the biogeochemical material for sampling and the

\footnotetext{
${ }^{1}$ Departamento de Ciências da Terra, Faculdade de Ciências e Tecnologia, Universidade de Coimbra, 3000-272 Coimbra; jpratas@dct.uc.pt

${ }^{2}$ Departamento de Geologia, Universidade de Trás-os-Montes e Alto Douro, 5001-801 Vila Real; pjcf@utad.pt

${ }^{3}$ Centro de Geociências da Universidade de Coimbra
} 
interpretation of the analytical results that takes into account the physiological mechanisms of plant adaptation and, in particular, the pedogeochemical phenomena. In this work, the species present in soils and tailings with important W, As and some Cu contamination in the old mining area of Fragas do Cavalo (Oleiros, Castelo Branco, Central Portugal) have been characterised. The species were selected envisaging their use as bioindicators, meaning that they are able to detect the pollution present in soils for use in mineral exploration; and seeking their potential use in the restoration with metalotolerant species able to tolerate the geochemical stress imposed by these conditions.

Keywords - Bioaccumulation, bioindicating, heavy metals, arsenic, revegetation

\section{1 - Introduçáo}

A utilização de espécies vegetais na prospecção de contaminaçôes, naturais ou induzidas por atividades antropogénicas, pressupóe o conhecimento da sua capacidade de utilização e retenção dos elementos presentes no substrato. A composição química duma planta é função de um grande número de factores que atuam em simultâneo. É necessário distinguir entre factores internos (fisiológicos), resultantes da atividade metabólica das próprias plantas e externos (ecológicos), relacionados com o habitat, que inclui factores geológicos, pedológicos e climáticos.

As mesmas bases de estudo podem também ser aplicadas na redução de impactes ambientais devido a contaminantes químicos por metais pesados e metaloides, através da seleção de espécies capazes de utilizarem, em grande escala, esses elementos, os quais podem ser incorporados nos seus tecidos. Numa outra perspectiva, poderão apresentar grande importância na minimização de impactes as espécies cujos mecanismos internos impedem a absorção de elementos tóxicos, devido à sua retenção no sistema radicular ou em fungos simbiontes associados, os quais podem complexar os metais do solo, evitando níveis mais elevados de toxicidade. Na recuperação ambiental de áreas mineiras parece-nos que este tipo de abordagem deverá sempre ser tido em consideração.

\section{2 - Área de estudo}

A zona das minas de Fragas do Cavalo encontra-se na Serra de Alvelos, no concelho de Oleiros e dista cerca de $80 \mathrm{~km}$ para Oeste de Castelo Branco e $90 \mathrm{~km}$ para Este de Coimbra (Fig. 1). Embora enquadrada em zona do pinhal, devido aos constantes incêndios florestais a vegetação aqui é a característica das zonas montanhosas de altitude moderada do centro do país, sem coberto vegetal arbóreo significativo. Está englobada no Centro-Este-montanhoso segundo a divisão fitogeográfica (FRANCO, 1984). Encontra-se na área definida como de clima húmido com um índice hídrico de 60 a 100\% (FRANCO \& AFONSO, 1982); uma humidade relativa do ar anual média menor que $65 \%$, uma precipitação anual de 1200 a $1400 \mathrm{~mm}$, uma temperatura média anual de 7,5 a $10{ }^{\circ} \mathrm{C}$ e uma insolação de 2300 a 2400 horas por ano (ATLAS DO AMBIENTE, 1981). 

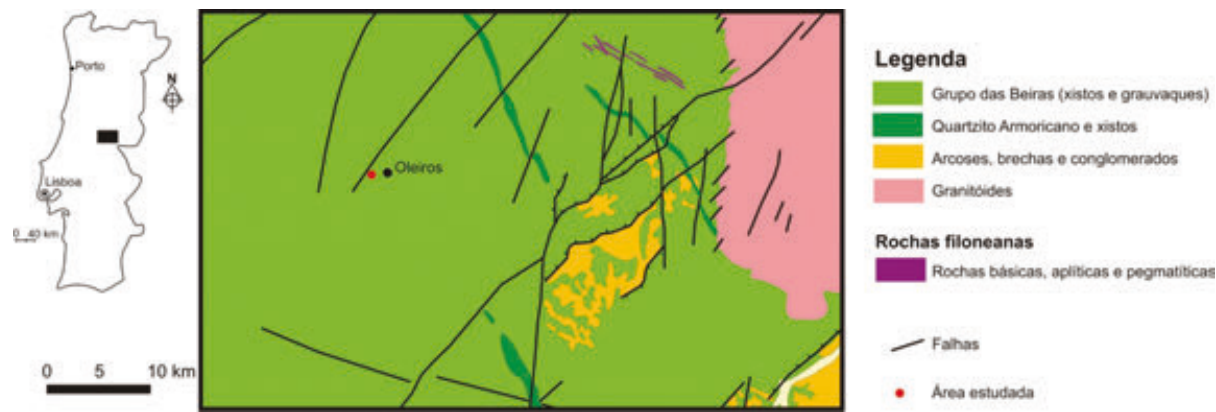

Fig. 1 - Enquadramento geográfico e geológico das minas de Fragas do Cavalo (adaptado da Carta Geológica de Portugal, à escala 1/500000, editada pelos Serviços Geológicos de Portugal, 1992).

A mineralização está encaixada em rochas metassedimentares do Grupo das Beiras (Complexo Xisto-Grauváquico) numa unidade constituída quase unicamente por xistos com alguns níveis, pouco espessos, de siltitos; é composta essencialmente por volframite, arsenopirite, pirite e calcopirite. Está relacionada com uma cúpula granítica não aflorante, mas evidenciada por uma zona com disposição circular de xistos mosqueados. Os filóes mineralizados apresentam uma direcção E-W e forte pendor para N. Na extremidade W, a estrutura filoneana foi objeto de exploração mineira, apresenta-se cortada por falhas N-S com uma importante componente horizontal e com movimentação direita.

A cúpula granítica deve estar relacionada com intrusões hercínicas que se desenvolvem para leste desta área, na região de Castelo Branco, e corresponderia a uma mineralização análoga à das minas da Panasqueira. Todavia o afloramento de granito mais próximo situa-se a cerca de $3 \mathrm{~km}$ para noroeste desta mineralização, correspondendo a um granito Pré-câmbrico - Granito de Álvaro, semelhante aos do Coentral e Pedrógão, mas com uma auréola de escarnitos com scheelite (CONDE \& PRATAS, 1993).

\section{3 - Métodos e Técnicas}

\section{1 - Amostragem}

$\mathrm{Na}$ área de estudo foi efetuado um perfil transversal à zona mineralizada e escombreiras. Sobre este perfil, e em intervalos de 20 metros foram efetuadas colheitas de solos e das espécies vegetais presentes, num círculo com raio de cerca de 2 metros e centrado em cada ponto de amostragem, orientado de modo a que cerca de $1 / 3$ do total de amostras se situassem na área contaminada. O total dos pontos de amostragem foi de 21. A metodologia adoptada seguiu as orientaçôes definidas por KOVALEVSKII (1979) \& BROOKS (1983).

Os trabalhos efetuados envolveram um menor tempo possível na sua execução (1 dia), por forma a evitar variações temporais na utilização dos diferentes elementos químicos pelas plantas. 
As amostras dos solos foram colhidas sempre no horizonte B, por forma a minimizar a influência da matéria orgânica contida em horizontes menos profundos. A profundidade de colheita foi variável atendendo a que se trata de solos de montanha muito pouco desenvolvidos. A quantidade de material colhido rondou 500 gramas. Esse material, constituiu uma homogeneização das colheitas de cerca de 4 pontos, dentro do círculo proposto, em torno de cada ponto de amostragem.

A amostragem do material vegetal envolveu a colheita de todas as espécies presentes na área envolvente dos pontos de amostragem, por forma a obter, tanto quanto possível, uma quantidade de material suficiente para a execução de todas as análises propostas. O material amostrado incidiu sobre a parte aérea, dada a economicidade que representa nos levantamentos biogeoquímicos.

\section{2 - Preparaçáo das amostras e análises químicas}

As amostras dos solos foram secas na estufa, a $60{ }^{\circ} \mathrm{C}$, após o que foram crivadas abaixo de 60 malhas (FLETCHER, 1981; BROOKS, 1983; PRATAS et al., 2005; FAVAS et al., 2011). Esta fracção foi posteriormente moída abaixo de 200 malhas para as análises químicas.

O material vegetal foi abundantemente lavado em água corrente e depois em água destilada. Após a lavagem, foi seco em estufa a $60{ }^{\circ} \mathrm{C}$ durante algumas horas, tendo sido seguidamente moído, em moinho de corte próprio para o efeito, por forma a obter a sua homogeneização (FLETCHER, 1981; BROOKS, 1983; VAN LOON, 1985; PRATAS et al., 2005).

As análises dos solos foram feitas por espectrofotometria de absorção atómica (EAA), simples ou acoplada a sistema de geração de hidretos, e técnicas colorimétricas, variáveis para os diferentes elementos. Assim, para as análises químicas de $\mathrm{Ag}, \mathrm{Co}, \mathrm{Cr}, \mathrm{Cu}, \mathrm{Fe}$, $\mathrm{Mn}, \mathrm{Ni}, \mathrm{Pb}$ e Zn, utilizou-se a EAA, após uma digestâo ácida das amostras com $\mathrm{HNO}_{3}$, $\mathrm{HClO}_{4}$ e HF, conforme a metodologia sugerida por LUCAS \& SEQUEIRA (1971). Para a análise de As e $\mathrm{Sn}$, utilizou-se o gerador de hidretos acoplado à EAA, partindo da solução proveniente do ataque ácido referido anteriormente e seguindo a metodologia sugerida por ASLIN (1976), VIJAN et al. (1976), FLETCHER (1981) \& VAN LOON (1985), e conforme os métodos e técnicas recomendados pelo manual de operação de geração de hidretos da PERKIN ELMER (1985). Para a análise do W, utilizou-se o método colorimétrico descrito por QUIN \& BROOKS (1972).

Relativamente ao material vegetal, os processos analíticos envolveram a incineração, salvo para a análise de As onde a maceração foi efetuada por via húmida, tendo sido utilizadas as mesmas técnicas que envolveram a análise dos solos. Não foi analisado o Sn no material vegetal devido às reduzidas concentraçóes encontradas nos solos.

\section{3 - Análise de dados}

A análise dos dados envolveu o estabelecimento de correlaçóes lineares e a análise em componentes principais (ACP). Para a sua execução partimos do pressuposto de que as distribuiçóes apresentadas pelos dados de que dispomos seguem todas a lei normal, pois, 
não sendo possível ajustar leis diferenciadas para cada subconjunto de dados, a hipótese da multi-normalidade surge como uma aproximação adequada que fundamenta os tratamentos paramétricos efetuados.

A utilização da ACP no presente trabalho visou, fundamentalmente, estabelecer as relaçóes multi-dimensionais entre as diversas variáveis de modo a detectar as associaçôes de elementos responsáveis pelas contaminaçôes e a sua atuação sobre as espécies vegetais presentes nas diferentes áreas. Com base na metodologia sugerida por PRATAS et al. (2005) foi também calculado o Índice Absoluto da Anomalia Biogeoquímica (IAAB) e o Índice Relativo da Anomalia Biogeoquímica (IRAB). Além disso, pretendeu-se separar os pontos que manifestam anomalia no solo daqueles que pertencem ao fundo regional.

\section{4 - Selecção de espécies}

A seleção das espécies bioindicadoras obedeceu aos critérios definidos para a obtenção dos índices indicadores anteriormente referidos (PRATAS et al., 2005). Para a definição das espécies inibidoras e acumuladoras utilizaram-se os critérios referidos por KOVALEVSKII (1979) e BROOKS (1983), relativamente à definição de barreiras fisiológicas, modificados por PRATAS (1996). As espécies inibidoras são aquelas que apresentam barreiras fisiológicas à assimilação e/ou acumulação de metais pesados nos seus tecidos, enquanto que as acumuladoras permitem o "uptake" de metais pesados e a acumulação destes nos seus tecidos com concentraçôes variáveis.

\section{4 - Resultados}

\section{1 - Solos}

Os resultados analíticos obtidos para solos, que se resumem na Tabela 1, mostram que, dos elementos presentes na mineralização, são o As, Cu e W aqueles que fornecem anomalias mais notórias. Por outro lado, a fraca concentração de Mn nos solos, está de acordo com o facto de a volframite presente na mineralização se aproximar mais do termo ferberítico.

Tabela 1 - Resumo dos dados analíticos obtidos nos solos (valores em $\mathrm{mg} / \mathrm{kg}$, excepto o Fe que é expresso em $\% ; \mathrm{n}=21)$.

\begin{tabular}{cccccccccccccc}
\hline & $\mathrm{pH}$ & $\mathrm{Ag}$ & $\mathrm{As}$ & $\mathrm{Co}$ & $\mathrm{Cr}$ & $\mathrm{Cu}$ & $\mathrm{Fe}$ & $\mathrm{Mn}$ & $\mathrm{Ni}$ & $\mathrm{Pb}$ & $\mathrm{Sn}$ & $\mathrm{W}$ & $\mathrm{Zn}$ \\
\hline Mínimo & 4,2 & 0,67 & 23,7 & 2,47 & 53,3 & 23,0 & 2,62 & 62,5 & 5,04 & 22,6 & 1,10 & 1,59 & 48,1 \\
Máximo & 5,1 & 1,42 & 2045 & 12,8 & 85,5 & 296 & 7,57 & 392 & 23,2 & 193 & 4,85 & 990 & 77,2 \\
Média & 4,6 & 0,90 & 324 & 8,30 & 72,0 & 77,3 & 3,86 & 155 & 16,1 & 42,1 & 2,9 & 80,0 & 63,5 \\
D.P. & 0,2 & 0,20 & 552 & 2,74 & 7,63 & 70,5 & 1,05 & 88 & 4,90 & 35,1 & 1,2 & 210 & 8,98 \\
\hline
\end{tabular}

Os dados obtidos foram submetidos à ACP, para permitir verificar as relaçóes existentes, de modo a clarificar as oposiçóes e similitudes entre eles. 
Os resultados obtidos mostram que os dois primeiros eixos explicam cerca de $60 \%$ da variância total e que o terceiro eixo, embora com uma certa quantidade de explicaçáo, apenas é explicado pelo comportamento do Sn. Assim, em virtude da ausência deste elemento na mineralização e dos seus baixos teores, optou-se apenas pela utilização dos dois primeiros eixos. Relativamente à contribuição dos vários elementos para a formação dos referidos eixos, verifica-se que, para o eixo 1, os elementos que mais o caracterizam são, por ordem decrescente de importância, $\mathrm{Cu}, \mathrm{As}, \mathrm{W}, \mathrm{Fe}$ e $\mathrm{Ag}$ e, por oposição, o Ni, enquanto que para o eixo 2 são o $\mathrm{Zn}, \mathrm{Mn}$, Cr e Co. O Sn contribui apenas para a formação do eixo 3, enquanto que o eixo 4 é caracterizado pelo $\mathrm{Pb}$ e, em oposiçáo, pelo $\mathrm{pH}$.

Ao fazer a projeção dos elementos no plano fatorial definido pelos eixos 1 e 2 (Fig. 2), nota-se uma grande oposiçáo dos elementos constitutivos da mineralização em relaçáo aos restantes elementos, observando-se, também, que os elementos de fuga, como o são o $\mathrm{Ni}$, Co e Cr, denotam uma maior oposição em relação à mineralização.

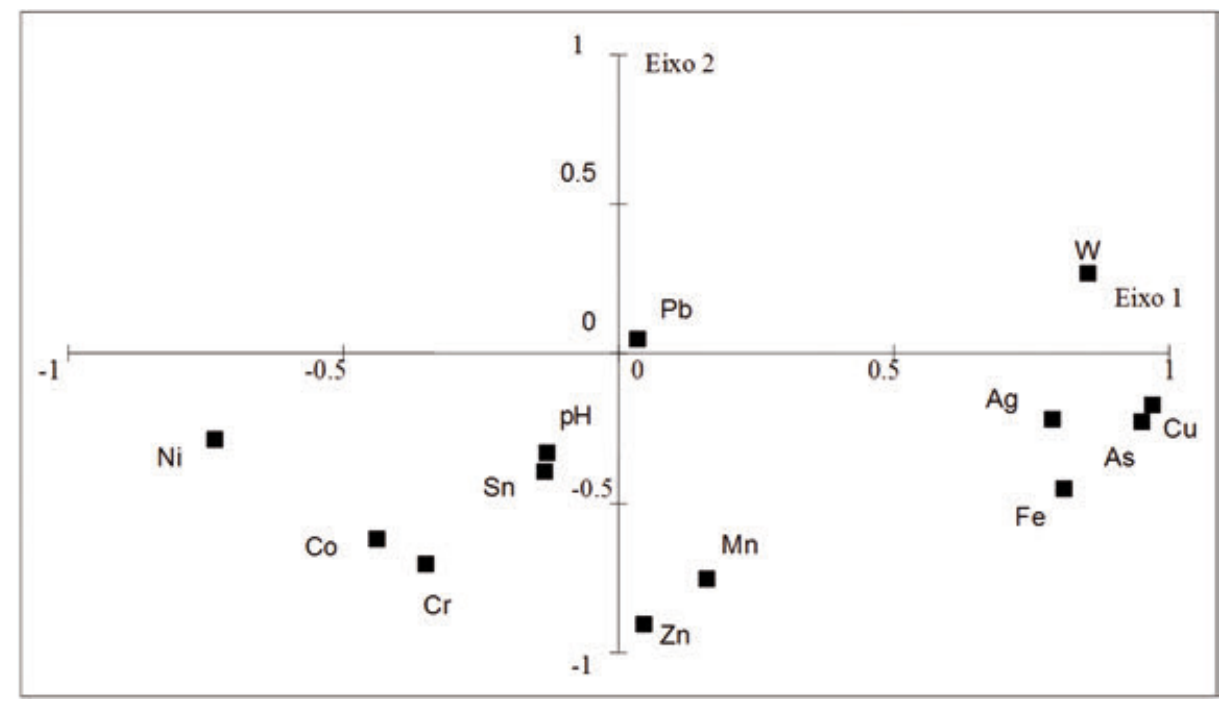

Fig. 2 - Projeção dos elementos nos eixos 1 e 2 da ACP.

\section{2 - Plantas}

$\mathrm{Na}$ área de estudo as espécies vegetais representativas são pouco diversificadas, não só por se tratar de áreas montanhosas com pequeno grau de intervenção mas também devido aos fogos florestais que provocaram a degradação do coberto arbóreo.

As espécies analisadas incluíram: Erica australis, Erica umbellata, Pterospartum tridentatum, Halimium ocymoides, Arbutus unedo, Cistus salvifolius, Hypochaeris radicata, Pinus pinaster (jovens), Anarrhinum bellidifolium, Conyza canadensis, Andryala integrifolia, Agrostis delicatula e Agrostis curtisii. Algumas destas espécies apresentavam uma área de distribuição bastante restrita e apenas foram consideradas na análise de dados as seis primeiras das espécies atrás referidas. Os resultados estão resumidos na Tabela 2. 
De salientar que os resultados apresentados se referem apenas aos elementos químicos formadores da anomalia, ou seja, os que caracterizam a contaminação.

Tabela 2 - Resultados analíticos obtidos para o material vegetal (valores médios).

\begin{tabular}{lccccc}
\hline \multicolumn{1}{c}{ Espécies de plantas } & $\mathrm{n}$ & $\mathrm{As}$ & $\mathrm{Cu}$ & $\mathrm{Fe}$ & $\mathrm{W}$ \\
\hline Erica australis & 20 & 0,13 & 3,85 & 157 & 0,16 \\
Erica umbellata & 16 & 0,42 & 4,47 & 104 & 0,36 \\
Pterospartum tridentatum subsp. lasianthum & 19 & 0,18 & 2,83 & 85 & 0,16 \\
Halimium ocymoides & 19 & 0,36 & 4,17 & 122 & 0,24 \\
Arbutus unedo (folhas) & 9 & 0,29 & 3,34 & 88 & 0,38 \\
Arbutus unedo (caules) & 9 & 0,17 & 2,92 & 122 & 0,27 \\
Cistus salvifolius & 8 & 0,52 & 4,45 & 61 & 0,36 \\
\hline
\end{tabular}

Nenhuma das espécies foi encontrada em todos os pontos de amostragem, não estando essa ausência relacionada com a presença ou não da contaminação do solo mas apenas com factores de heterogeneidade do coberto vegetal.

Com base na globalidade dos dados obtidos procedeu-se ao cálculo do índice da anomalia biogeoquímica (PRATAS et al., 2005), estando os resultados referidos na Tabela 3.

Tabela 3 - Índices da anomalia biogeoquímica para as espécies representativas da área.

\begin{tabular}{lcccc}
\hline \multicolumn{1}{c}{ Espécies de plantas } & As & $\mathrm{Cu}$ & $\mathrm{Fe}$ & $\mathrm{W}$ \\
\hline Erica australis & 15,4 & 1,9 & 0 & 9,7 \\
Erica umbellata & 3,6 & 0,7 & 0 & 28,5 \\
Pterospartum tridentatum subsp. lasianthum & 6,4 & 1,3 & 0 & 10,8 \\
Halimium ocymoides & 8,8 & 2,6 & 0 & 7,3 \\
Arbutus unedo (folhas) & 10,3 & 0,4 & 0 & 29,5 \\
Arbutus unedo (caules) & 0 & $-2,8$ & 0 & 19,4 \\
Cistus salvifolius & 9,1 & 0 & 0 & 5,5 \\
\hline
\end{tabular}

Os resultados obtidos mostram que para o Fe nenhuma das espécies que foram sujeitas a tratamento de dados têm capacidade de formar anomalia; para o $\mathrm{Cu}$ todas as espécies apresentam uma anomalia bastante reduzida, nula ou mesmo negativa mostrando antagonismo deste elemento no Arbutus unedo relativamente aos outros elementos; para o As todas as espécies apresentam anomalia de intensidade variável, embora mais intensa na Erica australis e nas folhas do Arbutus unedo e nula para os ramos desta última espécie; para o W é a Erica umbellata e o Arbutus unedo (tanto em folhas como em ramos) que apresentam anomalia mais intensa, embora todas as espécies reflitam anomalia.

Os resultados obtidos apenas se referem à anomalia produzida nas espécies vegetais sem ter em conta a intensidade da anomalia produzida nos solos. Atendendo também a que as espécies referidas não foram colhidas em todos os pontos, nem nos mesmos pontos de amostragem, foi necessário proceder-se a uma normalização dos resultados que tenha em conta a anomalia produzida nos solos subjacentes. Para comparar esta nova componente, calcularam-se os índices relativos da anomalia biogeoquímica referidos na Tabela 4. 
Tabela 4 - Índices relativos da anomalia biogeoquímica.

\begin{tabular}{lcccc}
\hline \multicolumn{1}{c}{ Espécies de plantas } & $\mathrm{As}$ & $\mathrm{Cu}$ & $\mathrm{Fe}$ & $\mathrm{W}$ \\
\hline Erica australis & 0,36 & 0 & 0 & 0,14 \\
Erica umbellata & 0,24 & 0 & 0 & 0,39 \\
Pterospartum tridentatum subsp. lasianthum & 0,23 & 0 & 0 & 0,10 \\
Halimium ocymoides & 0,31 & 0,31 & 0 & 0,09 \\
Arbutus unedo (folhas) & 0,33 & 0,03 & 0 & 0,37 \\
Arbutus unedo (caules) & 0 & 0 & 0 & 0,21 \\
Cistus salvifolius & 0,24 & 0 & 0 & 0,07 \\
\hline
\end{tabular}

Este novo índice permite verificar que para o $\mathrm{Cu}$ apenas o Halimium ocymoides reflete a anomalia produzida no solo e muito reduzidamente as folhas do Arbutus unedo; o As do solo influencia as concentraçóes observadas na planta para qualquer das espécies consideradas, embora com maior intensidade para a Erica australis, Arbutus unedo (folhas) e Halimium ocymoides, não sendo possível a sua identificação a partir dos ramos do Arbutus unedo; o W reflete a contaminação dos solos principalmente na Erica umbellata e Arbutus unedo.

Considerando agora as barreiras fisiológicas ao nível da parte aérea, em função da razão entre o teor máximo e o teor de background, parâmetros básicos para a definição do tipo de barreiras fisiológicas, e considerando como teor de background a média das concentraçóes obtidas para as amostras consideradas do fundo biogeoquímico e separadas a partir da ACP, conduziram aos valores da Tabela 5.

Tabela 5 - Razão entre o teor máximo e de background para as espécies vegetais.

\begin{tabular}{llllc}
\hline \multicolumn{1}{c}{ Espécies de plantas } & $\mathrm{As}$ & $\mathrm{Cu}$ & $\mathrm{Fe}$ & $\mathrm{W}$ \\
\hline Erica australis & 5,9 & 1,6 & 2,6 & 4,2 \\
Erica umbellata & 3,3 & 1,4 & 1,6 & 10,5 \\
Pterospartum tridentatum subsp. lasianthum & 6,4 & 1,8 & 1,7 & 11,4 \\
Halimium ocymoides & 8,1 & 2,2 & 2,6 & 3,2 \\
Arbutus unedo (folhas) & 3,8 & 1,5 & 1,4 & 5,2 \\
Arbutus unedo (caules) & 1,7 & 1,4 & 2,5 & 4,0 \\
Cistus salvifolius & 4,5 & 1,5 & 1,5 & 6,0 \\
\hline
\end{tabular}

Porém se se considerar o grau de representatividade dos coeficientes de correlação linear da relação planta-solo chega-se aos resultados constantes da Tabela 6.

Tabela 6 - Representatividade dos coeficientes de correlação linear entre as concentraçóes no solo e na planta.

\begin{tabular}{lcccc}
\hline \multicolumn{1}{c}{ Espécies de plantas } & As & $\mathrm{Cu}$ & $\mathrm{Fe}$ & W \\
\hline Erica australis & $\mathrm{S}^{* *}$ & $\mathrm{NS}$ & $\mathrm{NS}$ & $\mathrm{S}^{* *}$ \\
Erica umbellata & $\mathrm{S}^{*}$ & $\mathrm{NS}$ & $\mathrm{NS}$ & $\mathrm{S}^{* *}$ \\
Pterospartum tridentatum subsp. lasianthum & $\mathrm{S}^{* *}$ & $\mathrm{PS}$ & $\mathrm{NS}$ & $\mathrm{S}$ \\
Halimium ocymoides & $\mathrm{S}$ & $\mathrm{S}$ & $\mathrm{NS}$ & $\mathrm{S}$ \\
Arbutus unedo (folhas) & $\mathrm{S}^{* *}$ & $\mathrm{NS}$ & $\mathrm{NS}$ & $\mathrm{S}$ \\
Arbutus unedo (caules) & $\mathrm{NS}$ & $\mathrm{NS}$ & $\mathrm{NS}$ & $\mathrm{PS}$ \\
Cistus salvifolius & $\mathrm{PS}$ & $\mathrm{NS}$ & $\mathrm{NS}$ & $\mathrm{PS}$ \\
\hline
\end{tabular}

Nota: Os símbolos S**, S*, S, PS e NS representam respectivamente graus de confiança $>99.9 \%$, de 99.0 a $99.9 \%$, de 95 a $99 \%$, de 90 a $95 \%$ e $<90 \%$ 
Considerando tanto a representatividade da relação planta-solo como da razão entre o teor máximo e teor de background verifica-se que as espécies analisadas apresentam-se com médias barreiras fisiológicas para o As e W e com baixas barreiras para o $\mathrm{Cu}$ e o Fe.

Apesar dos resultados observados para o $\mathrm{Cu}$ no Halimium ocymoides permitirem verificar uma boa relação entre os teores presentes na planta e os observados no solo a amplitude dos teores observados nâo permitem que seja classificada como médias barreiras. O facto de não permitir retirar outras conclusóes que não esta deve-se ao facto de nesta área, apesar do $\mathrm{Cu}$ ser um elemento acompanhante da mineralização e formador de contaminação, a sua concentração não é suficientemente elevada de modo a produzir outro tipo de comportamento.

\section{5 - Conclusóes}

Relativamente à capacidade de utilização das espécies analisadas relativamente a campanhas de prospecção biogeoquímica de contaminaçóes geradas por este tipo de paragénese verifica-se, em função dos elementos químicos considerados e por ordem decrescente de importância, que as espécies mais indicadas são: para o As - Erica australis, Arbutus unedo (folhas), Halimium ocymoides, Erica umbellata, Cistus salvifolius e Pterospartum tridentatum; para o $\mathrm{Cu}$ - Halimium ocymoides; para o W - Erica umbellata, Arbutus unedo (folhas e ramos), Erica australis, Pterospartum tridentatum, Halimium ocymoides e Cistus salvifolius.

Quanto à possibilidade de utilização das espécies na revegetação de áreas com este tipo de contaminaçôes, verifica-se que todas as espécies se apresentam com condiçóes de acumularem nos seus tecidos os principais elementos causadores da contaminação (As e W), embora se apresentem apenas com médias barreiras fisiológicas, não implicando portanto uma alta entrada destes elementos no ecossistema.

\section{Referências Bibliográficas}

ASLIN, G.E.M. (1976) The determination of arsenic and antimony in geological materials by flameless atomic absorption espectrometry, J. Geochem. Explor. 6: 321-330.

ATLAS DO AMBIENTE (1981) Agência Portuguesa do Ambiente. Disponível em: http://sniamb.apambiente. pt/webatlas/.

BROOKS, R.R. (1983) Biological Methods of Prospecting for Minerals, John Willey \& Sons, New York, 322.

CONDE, L.E.N. \& PRATAS, J.A. (1993) The precambrian granite of Álvaro (Central Portugal) and the associated tungsten mineralization, Terra Cognita - E.U.G., 156.

FAVAS, P.J.C.; PRATAS, J.; GOMES, M.E.P. \& CALA, V. (2011). Selective chemical extraction of heavy metals in tailings and soils contaminated by mining activity: Environmental implications. J. Geochem. Explor. 111: 160-171.

FLETCHER, W.K. (1981) Analytical Methods in Geochemical Prospecting, Handbook of Exploration Geochemistry, Vol.1, Govett, G.J.S, Ed., Elsevier, 255.

FRANCO, J.A. (1984) Nova Flora de Portugal (Continente e Açores), Vol. II, Clethraceae - Compositae, Lisboa, 659. 
FRANCO, J.A. \& AFONSO, M.L.R. (1982) Distribuição de Pteridófitos e Gimnospérmicas em Portugal, col. Parques Naturais, 14, Serviço Nacional de Parques, Reservas e Património Paisagístico, Lisboa, 327.

KOVALEVSKII, A.L. (1979) Biogeochemical Exploration for Mineral Deposits, Oxonian Press PVT., Nova Deli, 136.

LUCAS, M.D. \& SEQUEIRA, E.M. (1971) Determinações de alumínio, cálcio, cobalto, cobre, ferro, magnésio, manganésio, potássio, sódio, titânio e zinco totais nos solos por ataque nítrico-perclórico-fluorídrico e por absorção atómica e emissão de chama, Pedologia, Oeiras, 6, 55-66.

PERKIN-ELMER (1985) Mercury/Hydride System, Operator's Manual.

PRATAS, J.A.M.S. (1996) Aplicações de Prospecção Biogeoquímica. Selecção de espécies bioindicadoras em algumas áreas mineiras de Portugal. Tese de Doutoramento, 3 Vol., Universidade de Coimbra, 1027.

PRATAS, J.; PRASAD, M.N.V,; FREITAS, H. \& CONDE, L. (2005). Plants growing in abandoned mines of Portugal are useful for biogeochemical exploration of arsenic, antimony, tungsten and mine reclamation. J. Geochem. Explor. 85: 99-107.

QUIN, B.F. e BROOKS, R.R. (1972) The rapid determination of tungsten in soils, rocks and vegetation, Anal. Chim. Acta, 58: 301-309.

VAN LOON, J.C. (1985) Selected Methods of Trace Metal Analysis: Biological and Environmental Samples, John Willey \& Sons., 355.

VIJAN, P.N., RAYNER, A.C., STURGIS, D. \& WOOD, G.R. (1976) A semi-automated method for the determination of arsenic in soil and vegetation by gas-phase sampling and atomic absorption spectrometry, Anal. Chim. Acta, 82: 329-336. 